\title{
Identification the ferroptosis-related gene signature in Gastric cancer based on weighted gene co- expression network analysis (WGCNA)
}

Feng Wang ( $\square$ youhe5@163.com )

People's Hospital of binhai

\section{Cheng Chen}

The people' s hospital of Binghai country

Wei-Peng Chen

The people's hospital of Binghai country

Zu-Ling Li

The people's hospital of Binghai country

Hui Cheng

The people's hospital of Binghai country

\section{Research Article}

Keywords: Gastric cancer, Ferroptosis, WGCNA, Prognostic model

Posted Date: May 13th, 2021

DOl: https://doi.org/10.21203/rs.3.rs-486587/v1

License: (c) (1) This work is licensed under a Creative Commons Attribution 4.0 International License. Read Full License 


\section{Abstract \\ Background}

Ferroptosis is a mode of regulated cell death that depends on iron, plays pivotal roles in regulating various biological process in human cancers. However, the role of ferroptosis in Gastric cancer (GC) remains unclear.

\section{Methods}

A total of 2721 differentially expressed genes (DEGs) were filtered base on The Cancer Genome Atlas (TCGA) $(n=375)$ dataset. Gene modules were identified based on co-expression network analysis (WGCNA). Functional analysis was performed to explore the biological function. Lasso-penalized and univariate Cox regression (UCR) analysis, survival genes were screened out to construct a prognostic model, which validated by the GSE43292 dataset. Gene set enrichment analysis (GSEA) for prognostic index was performed. Finally, the correlations of ferroptosis and immune cells were assessed through the TIMER database.

\section{Results}

Compared to normal specimens, 1063 highly upregulated and 1658 downregulated genes respectively and their normal counterparts in GC specimens were screened. WGCNA analysis was used and identified 7 modules, of which, blue module with the most significant enrichment result was selected. By taking intersections of blue module and differentially expressed ferroptosis-related genes (DEFRGs), we got 23 common genes. Functional analysis was performed to explore the biological function of the interested genes, and with the consequences Lasso-penalized and univariate Cox regression (UCR) analysis, survival genes were screened out to construct a prognostic model based on 3 genes (SLC1A5, ANGPTL4, and CGAS), which could play a role in predicting the survival of GC patients. UCR and multivariate Cox regression (MCR) analysis revealed that the prognostic index could be used as independent prognostic indicators and validated using another GSE84437 dataset. Notably, patients in high-risk groups had higher levels of higher mutation frequencies such as TTN and TP53.Mechanistically. Gene set enrichment analysis (GSEA) unveiled several significant and pathways involved in GC. TIMER analysis demonstrated that risk score strongly correlated with Macrophage and CD4 + T cells infiltration. In addition, high- and low-risk group illustrated different distributions in different immune status.

\section{Conclusions}

In this study, a novel FRGs signature was built. It could accurately predict GC prognosis and pave the new way for diagnosis and therapy strategy. May reflect the status of tumor immune microenvironment. 


\section{Introduction}

As a major public health issue globally, gastric cancer (GC) is the third leading cause of cancer-related death [1]. Due to early stages of GC are usually asymptomatic, and if patients are diagnosed at advanced stage, leading to poor survival [2]. Moreover, among patients with GC who receive adjuvant therapy, 50\% experience local or distant disease recurrence [3]. Hence, identification of reliable diagnostic and prognostic biomarkers is critical for GC, not only to improve prognostication but also to provide novel therapeutic targets for GC.

Ferroptosis is a novel form of regulated cell death that was described in 2012 [4]. It is a type of regulated cell death that is driven by intracellular lipid peroxidation and is highly dependent on reactive oxygen species (ROS) generation and iron availability [5]. Ferroptosis plays an important role in various tumors cells such as fibrosarcoma, lung cancer, and prostate cancer cells [6-8]. In addition, Several publications have reported that natural active components alleviated multidrug resistance of cancer and inhibit the progression of multiple tumors through inducing ferroptosis [9]. These findings suggest ferroptosis as a new player that regulates the tumor suppressive function. Nevertheless, prognostic models for FRGs have not been constructed for prediction of overall survival (OS) in GC patient.

The present study performed comprehensive analyses utilizing TCGA and GEO. We evaluated the prognostic value of the DEFRGs and constructed a three-mRNA signature that could effectively predict patient survival in TCGA dataset, and further validated in GEO dataset. Further, we further conducted functional studies on risk scores to elucidate the pathogenic mechanisms and provide scientific basis for clinical diagnosis and treatment.

\section{Materials And Methods}

\section{Patient Cohort and Data Preparation}

RNA-seq transcriptome data, the corresponding clinicopathological and copy number variation data were retrieved from TCGA database. (https://tcga-data.nci.nih.gov/tcga/) and GEO database (http://www.ncbi.nlm.nih.gov/geo/). TCGA data comprised 375 and 32 samples of GC tissues and adjacent normal tissues, respectively. By application of the GPL6947 platform (Illumina HumanHT-12 V3.0), GSE84437 contained 433 samples of GC tissues. The FRGs were obtained from other research [10], which downloaded FRGs from the FerrDb website (http://www.zhounan.org/ferrdb/) and PubMed (https://pubmed.ncbi.nlm.nih.gov/).

\section{Identifcation of DRGs and DFRGs}

Differential expression of DRGs and DFRGs between tumor and normal samples was assessed using the R-package called limma [11], The false discovery rate (FDR) $<0.05$ and $\mid \log 2$ (fold change) $\mid>0.5$, visualized on boxplots and heatmaps using the "ggpubr" and "heatmap" package, respectively. 


\section{Highly Coexpressed Gene Set-Gene Module}

The WGCNA was conducted by the WGCNA package [12] in R software. As a previous study showed that the WGCNA analysis was sensitive to batch effects and outlier samples, we performed hierarchical cluster analysis [13]. The module eigengene (ME), which is considered representative of the gene expression profiles, was calculated to identify clinical associated modules base on DRGs. To find the most tumor related modules, we conducted Module-Trait Relationships calculations for each module. Then, for genes in the significant tumor-related modules, we calculated the Gene Significance and Gene Module Membership (MM) within the genes, modules, and clinical traits. Finally, we identified the genes in the GC-related modules $[14,15]$.

\section{Acquisition of intersecting genes}

Overlapping genes were identified as candidates for the subsequent analysis and were oriented from the DFRGs and WGCNA analysis. The online tool Draw Venn Diagram

(http://bioinformatics.psb.ugent.be/webtools/Venn/). Coexpression analysis was performed using the "Corrplot" package.

\section{Function and pathway enrichment analyses}

The cluster Profiler package in R [16] was used to test the statistical enrichment of functions. To assess for functional categories, we used Gene ontology (GO) and Kyoto Encyclopedia of Genes and Genomes (KEGG) pathway. The P-value of $<0.05$ and q-value of $<0.05$ were set as the threshold.

\section{Construction of an FRGs-based risk score model}

The candidate FRGs were analyzed using UCR analysis $(p<0.05)$. The median values were defined as the cutoff values for high and low FRGs expression in Cox survival analysis. After identifying the prognosis related FRGs, we performed MCR analysis to identify independent prognostic FRGs. Finally, the risk score for each patient was calculated by taking the sum of Cox regression coefficient for each signature gene multiplied with its corresponding expression value. The immunohistochemistry staining of genes were examined by The Human Protein Atlas (HPA) (https://www.proteinatlas.org/about/download).

\section{Gene Set Enrichment Analysis (GSEA)}

was performed using GSEA v4.0.3 software with 1000 permutations and weighted enrichment statistics. The median risk score was used as the cut-off point for high or low-risk group classification [17].

\section{Immune Infiltration Analysis}

The infiltration level of immune cells in GC was predicted using the TIMER database [18]. Correlation analysis between six types of immune cells and risk score were then conducted (https://cistrome.shinyapps.io/timer/). 
Exploring Relationships Between Immune Components and risk groups

We used the CIBERSORT algorithm to estimate data on tumor-infiltrating immune cells. Violin plots were used to present the full distribution of the data [19].

\section{Statistical analysis}

For analysis of differences between two groups, Student's t test was performed. A Kaplan-Meier survival analysis was performed to estimate survival curve. Statistical analyses were performed using the statistical software $\mathrm{R}$ (version 4.0.2). A value less than $0.05(p<0.05)$ was considered significant.

\section{Results}

\section{Identifcation of DEGs}

After analysis, there was a total of 2721 DEGs between GC $(n=375)$ and normal samples $(n=32)$, including 1658 downregulated DEGs and 1063 upregulated DEGs. The heatmap and volcano plots are shown in Fig1a, b.

\section{Identification of significant gene modules by WGCNA}

Overall, 2721 DEGs and 407 samples were selected after gene and sample screening and preprocessing. We used a power calculation of $\beta=3$ (scale-free R2 $=0.895)($ Fig 2a). All selected gene dendrograms and their corresponding modules are displayed in Fig $2 \mathrm{~b}$. There were 7 modules, including the special gray module according to the network result (i.e., blue, black, red, brown, green, turquoise, and yellow modules). Among these 7 modules, red $(r=0.42 ; p=3 e-19)$, blue $(r=0.58 ; p=2 e-37)$, and black $(r=0.36 ; p=$ $1 \mathrm{e}-13)$ modules showed positive relationships with GC (Fig 1E). Furthermore, the genes in the turquoise and red modules showed strong negative correlations with GC (Brown: $r=-0.39 ; p=3 e-16$, Green: $r=$ $-0.4 ; p=1 \mathrm{e}-16$, Turquoise: $\mathrm{r}=-0.45 ; p=1 \mathrm{e}-21$, and Yellow: $\mathrm{r}=-0.31 ; p=9 \mathrm{e}-11$ ) (Fig 2c). Finally, we identified blue module as the key modules, in which there were 655 genes.

\section{The analysis of DEFRGs}

Next, we screened the DEFRGs in TCGA. We identified Sixty-one DEFRGs, including 31 up-regulated and 30 down-regulated genes (Fig 3; Table 1). Following, we compared the co-expressed genes in blue module with the DEFRGs, then a set of 23 shared genes were obtained (Fig 4a). Furthermore, a strong correlation among the FRGs (Fig 4b).

\section{Functional enrichment analysis}

To better understand the signaling pathways and functions of DEFRGs in ferroptosis, functional enrichment on the 23 genes were performed, and found that DEFRGs were enriched in iron-related pathways, such as regulation of cell aging, cell cycle arrest, and NF-kappaB binding. KEGG pathway 
analysis for the DEFRGs showed that genes involved in ferroptosis, including the Cellular senescence, p53 signaling pathway, Phenylalanine metabolism, HIF-1 signaling pathway and Cell cycle (Fig 5a, b).

\section{Construction of the three-gene-based GC prognostic model}

After that, UCR analysis of the screening results, including 23 FRGs, led to the identification of 5 FRGs as potential prognostic indicators of GC overall survival (OS), including ANGPTL4, SMPD1, MYB, SLC1A5, and CGAS (Fig 6). After primary filtering, we further shrink the scope of gene screening. Three genes were identified: SLC1A5, ANGPTL4, and CGAS. To establish an optimal prognostic gene model, MCR analysis was performed on the three genes. Risk score was calculated by the following formula: risk score $=$ [0.1497 $\times$ mRNA expression level of ANGPTL4] + [-0.1806 $\times$ mRNA expression level of SLC1 A5] + [-0.2385 $\times$ mRNA expression level of CGAS]. After calculating the risk score, we divided 370 patients into high-risk ( $n=185)$ and low-risk $(n=185)$ groups using median risk score as the cut-off. The patients in the high-risk group have worse OS than those in the low-risk group (Fig 7a). As the risk score rising, the patients had a shorter survival time, more death events (Fig7b, c). The risk heatmap showed the differences of three genes (SLC1A5, ANGPTL4, and CGAS) (Fig 7d). We used GEO group for further external validation of this 3-gene-based signature. We got the same result as above (Fig7e-h). Next, reliability and stability of the three gene-based model were further confirmed.

\section{Assessment of three FRGs Signature as Independent Prognostic Factor in GC Patients}

To further confirm whether the newly generated risk score was an independent risk factor in GC patients, we employed UCR and MCR analyses, which showed that T, N, metastasis and risk score were independent prognostic factors for OS in GC $(p<0.001)$ (Fig 8b, c). To evaluate the diagnostic performance of risk model in GC, ROC curves were constructed. The under the ROC (AUC) of risk score (0.611) was much higher than that of age (0.571), gender (0.539), T (0.590), N (0.572), and metastasis status (0.547) (Fig 8a). All results illustrated that the three FRGs signature was an independent prognostic factor in GC.

\section{Validation of Prognostic Performance of the FRGs Signature in GC}

To further assess outcome prediction, we combined the validation datasets (total of 433 patients) to evaluate the robustness of the three-gene signature. The results revealed the ROC curve AUC $=0.676$ for validation datasets (Fig 8d), which is similar to the one in the TCGA set. Cox regression analyses indicated that the risk score of the signature could be a powerful indicator of GC patient's clinical outcome (Fig $8 \mathrm{e}, \mathrm{f}$ ). Finally, the expressions of 3 signature genes were validated using the immunostaining results from the HPA database. As demonstrated in Fig 9, SLC1A5 was highly expressed in GC tissue, while ANGPTL4 and CGAS was downregulated in GC. Waterfall plot representing the mutant landscape of the top 30 in Fig 10. Interestingly, TTN[20], TP53[21], MUC16[22], and ARID1A[23] were the top mutations in both cohorts, which were involved in various biological processes. Besides, the frequencies of all mutated genes were higher in the high-risk group (96.74\%) (Fig 10a) than in the low-risk group (84.75\%) (Fig 10b), suggesting somatic mutation was positively correlated risk scores. 


\section{Immune Profile in risk groups}

Considering ferroptosis was strongly associated with immune status, the correlations between risk scores and immune status were further explored using CIBERSORT and TIMER to evaluate the immune cell features. As shown in Fig 10, Monocytes, Macrophages M2, Dendritic cells activated, Mast cells resting, and Neutrophils were up-regulated in the high-risk group, while T cells CD8, T cells CD 4 memory activated, T cells follicular helper, and Macrophages M2 were significantly down-regulated $(p<0.05)$ (Fig 11). Moreover, the levels of riskscore were positively associated with Macrophages $(\mathrm{r}=0.366 ; p=6.846 \mathrm{e}-13)$ and T cells CD4( $\mathrm{r}=0.135 ; p=0.010)$ (Fig 12). The results showed strong correlations between ferroptosisrelated risk model and the immune state of GC.

\section{Evaluation of pathways within Both High- and Low-Risk TCGA Cohorts}

GSEA was performed to identify gene sets differentially expressed in high and low risk groups from the MSigDB databases (c2.cp.kegg.v6.2.symbols.gmt). The cell cycle, P53, MAPK, Ubiquitin mediated proteolysis, and TGF- $\beta$ signaling pathways were among the most significantly correlated enriched pathways (Fig 13).

\section{Discussion}

Ferroptosis is a recently recognized form of regulated cell death that is characterized by lipid peroxidation $[24,25]$. Increasing evidence suggest that ferroptosis plays a powerful role in enabling malignancy features of tumor [26]. However, fewer studies reported on ferroptosis-related research in GC and the systematic analysis has not been elucidated yet.

In the present study, Results in this study 23 differently expressed FRGs between GC tissue and normal tissue were revealed by WCGNA and limma, and 5 FRGs out of them were of prognostic value. Three gene prognostic model (SLC1A5, ANGPTL4, and CGAS) was constructed in TCGA dataset, and validated in GEO test set.

Solute carrier family 1 member 5 (SLC1A5), also referred to as ASCT2, is a sodium channel which acts as a high-affınity glutamine transporter in tumor cells [27]. Inhibition of SLC1A5 impedes glutamine uptake, leading to disturbance of mTORC1 signaling and activation of autophagy and cancer cell growth $[28,29]$. Increased SLC1A5 expression has been documented in melanoma [30], neuroblastoma [31], and GC [32]. In precent research shown that miR-137 suppresses ferroptosis by targeting glutamine transporter SLC1A5 in melanoma [33]. Angiopoietin-Like 4 (ANGPTL4) is a member of the angiopoietin family and the members of which act as regulators of lipid and glucose metabolism [34]. ANGPTL4 is overexpressed in several types of cancers and are associated with poor clinical outcome $[35,36]$. In addition, ANGPTL4 induces ROS accumulation and subsequent ferroptosis, and high ANGPTL4 levels and anoikis resistance as novel and highly relevant oncogenic properties that can be targeted by inducing ferroptosis [37]. Cyclic GMP-AMP synthase (CGAS) is a cytosolic DNA sensor that activates innate immune responses. cGAS catalyzes the synthesis of cGAMP, which functions as a second messenger that binds 
and activates the adaptor protein stimulator of interferon genes (STING) to induce type I interferons (IFNs) and other immune modulatory molecules [38]. The expression of cGAS, which produces cGAMP for STING activation, facilitating the activation of antitumor CD8 + T cell responses [39]. Furthermore, 8hydroxy-2Х-deoxyguanosine (8-OHG) functions as a damage-associated molecular pattern (DAMP) during ferroptotic cell death to trigger STING1-dependent macrophage polarization, supporting pancreatic cancer initiation and progression [40]. These results indicate that three genes were closely related to tumor ferroptosis.

Considering the pivotal role of ferroptosis in the progress tumor-invading immune cells of cancers, we explored the diverse immune cell infiltrations between low- and high- risk cohorts. A recent investigation documented that resting memory CD4 T cells as one of the most enriched tumor-invasion immune cell in GC samples [41]. Studies have reported follicular helper T cells in tertiary lymphoid structures of numerous tumors, implying that they participate in generating effective, as well as sustained antitumor immune responses [42]. M1 macrophages are linked to antitumor activity, whereas M2 macrophages are associated with cancer progression and metastasis [41]. Herein, in the high-risk group had an elevated level of M2 macrophage. In contrast, the patients in the low-risk group had elevated proportions of M1 macrophages. The GSEA collection found that cell cycle, P53, MAPK, Ubiquitin mediated proteolysis, and TGF- $\beta$ signaling pathways were most enriched. Recent studies have demonstrated the significant role played p53 in the regulation of glucose metabolism, reactive oxygen species (ROS) responses and ferroptosis [43]. Acetylation-defective p53 mutants were shown to promote ferroptosis, an iron-dependent, oxidative and non-apoptotic form of cell death [43]. TGF- $\beta 1$ is the most important cytokine of Epithelialto-mesenchymal transition (EMT). It has been reported that tumor cells in a high state of oxidative stress typically exhibit EMT, under which tumor cells may resist apoptotic cell death and increase their sensitivity to ferroptosis [44]. The mitogen-activated protein kinase (MAPK) signaling pathway has also been found to be involved in ferroptosis initiation [45]. Inhibiting MAPK signaling protects lung cancer cells against ferroptosis [46]. These GSEA results gave a detailed description of the ways and methods by which the three-gene signature participates in GC's progress, which may benefit future precision medicine research.Our study found a novel, robust FRGs signature for GC. the three-FRGs signature can effectively make GC patient prognosis evaluation and utilizing ferroptosis may be the therapeutic targets. However, further studies that verify these results are required.

\section{Abbreviations}

TCGA: The Cancer Genome Atlas databases; GEO: Gene Expression Omnibus databases; GC: Gastric cancer; WGCNA: Co-expression network analysis; FRGs: Ferroptosis-related genes; DEGs: Differentially expressed genes; DEFRGs: Differentially expressed ferroptosis-related genes; GO: Gene ontology; KEGG: Kyoto Encyclopedia of Genes and Genomes; URC: Univariate Cox regression analyses; MCR: Multivariate Cox regression analyses GSEA: Gene set enrichment analysis.

\section{Declarations}


Acknowledgements: None.

\section{Authors' contributions}

FW and $\mathrm{HC}$ contributed to the conception and design of the study; FW collected data and wrote the manuscript; CC performed the data analysis and constructed the figures and tables; WP and ZL reviewed and revised the manuscript and were involved in the conception of the study. Additionally, HC was responsible for the organization, revision and submission of this manuscript. All authors read and approved the final manuscript.

\section{Availability of data and materials}

The datasets analyzed was acquired from The Cancer Genome Atlas (TCGA) database (https://portal.gdc.cancer.gov/) and GEO database (http://www.ncbi.nlm.nih.gov/geo/).

Funding: None.

\section{Ethics approval and consent to participate}

This was not applicable to this manuscript.

\section{Consent for publication}

Consent for publication was obtained from all participants.

\section{Competing interests}

The authors declare that they have no competing interests.

\section{References}

1. Torre LA, Bray F, Siegel RL, Ferlay J, Lortet-Tieulent J, Jemal A: Global cancer statistics, 2012. CA: a cancer journal for clinicians 2015, 65(2):87-108.

2. Hironaka S: Anti-angiogenic therapies for gastric cancer. Asia-Pacific journal of clinical oncology 2019, 15(4):208-217.

3. Niccolai E, Taddei A, Prisco D, Amedei A: Gastric cancer and the epoch of immunotherapy approaches. World journal of gastroenterology 2015, 21(19):5778-5793.

4. Dixon SJ, Lemberg KM, Lamprecht MR, Skouta R, Zaitsev EM, Gleason CE, Patel DN, Bauer AJ, Cantley AM, Yang WS et al: Ferroptosis: an iron-dependent form of nonapoptotic cell death. Cell 2012, 149(5):1060-1072.

5. Zhao Y, Hu X, Liu Y, Dong S, Wen Z, He W, Zhang S, Huang Q, Shi M: ROS signaling under metabolic stress: cross-talk between AMPK and AKT pathway. Molecular cancer 2017, 16(1):79. 
6. Skouta R, Dixon SJ, Wang J, Dunn DE, Orman M, Shimada K, Rosenberg PA, Lo DC, Weinberg JM, Linkermann A et al: Ferrostatins inhibit oxidative lipid damage and cell death in diverse disease models. Journal of the American Chemical Society 2014, 136(12):4551-4556.

7. Cao JY, Dixon SJ: Mechanisms of ferroptosis. Cellular and molecular life sciences : CMLS 2016, 73(11-12):2195-2209.

8. Friedmann Angeli JP, Schneider M, Proneth B, Tyurina YY, Tyurin VA, Hammond VJ, Herbach N, Aichler M, Walch A, Eggenhofer $E$ et al: Inactivation of the ferroptosis regulator Gpx4 triggers acute renal failure in mice. Nature cell biology 2014, 16(12):1180-1191.

9. Li QY, Wang F, Yang XC, Wan XM, Zhao TY, Chang FF, Du JL, Wang BB, Jia XY, Zhou XY: Effects of times and storage conditions of Duddingtonia flagrans chlamydospores in sodium alginate pellets on its nematode predatory ability.

10. Liu Y, Zhang X, Zhang J, Tan J, Li J, Song Z: Development and Validation of a Combined Ferroptosis and Immune Prognostic Classifier for Hepatocellular Carcinoma. 2020, 8(1716).

11. Ritchie ME, Phipson B, Wu D, Hu Y, Law CW, Shi W, Smyth GK: limma powers differential expression analyses for RNA-sequencing and microarray studies. Nucleic acids research 2015, 43(7):e47.

12. Langfelder $P$, Horvath S: WGCNA: an R package for weighted correlation network analysis. BMC bioinformatics 2008, 9:559.

13. Langfelder P, Horvath S: Fast R Functions for Robust Correlations and Hierarchical Clustering. Journal of statistical software 2012, 46(11).

14. Oldham MC, Konopka G, Iwamoto K, Langfelder P, Kato T, Horvath S, Geschwind DH: Functional organization of the transcriptome in human brain. Nature neuroscience 2008, 11(11):1271-1282.

15. Giulietti M, Occhipinti G, Principato G, Piva F: Weighted gene co-expression network analysis reveals key genes involved in pancreatic ductal adenocarcinoma development. Cellular oncology (Dordrecht) 2016, 39(4):379-388.

16. Yu G, Wang LG, Han Y, He QY: clusterProfiler: an R package for comparing biological themes among gene clusters. Omics : a journal of integrative biology 2012, 16(5):284-287.

17. Subramanian A, Tamayo P, Mootha VK, Mukherjee S, Ebert BL, Gillette MA, Paulovich A, Pomeroy SL, Golub TR, Lander ES et al: Gene set enrichment analysis: a knowledge-based approach for interpreting genome-wide expression profiles. Proceedings of the National Academy of Sciences of the United States of America 2005, 102(43):15545-15550.

18. Li T, Fan J, Wang B, Traugh N, Chen Q, Liu JS, Li B, Liu XSJCR: TIMER: A Web Server for Comprehensive Analysis of Tumor-Infiltrating Immune Cells. 2017, 77(21):e108.

19. Newman AM, Liu CL, Green MR, Gentles AJ, Feng W, Xu Y, Hoang CD, Diehn M, Alizadeh AAJNM: Robust enumeration of cell subsets from tissue expression profiles. 2015, 12(5):453-457.

20. Liu Z, Wang L, Guo C, Liu L, Jiao D, Sun Z, Wu K, Zhao Y, Han XJJoc, medicine m: TTN/OBSCN 'Double-Hit' predicts favourable prognosis, 'immune-hot' subtype and potentially better immunotherapeutic efficacy in colorectal cancer. 2021, 25(7):3239-3251. 
21. Chakraborty S, Martines C, Porro F, Fortunati I, Bonato A, Dimishkovska M, Piazza S, Yadav B, Innocenti I, Fazio R et al: B Cell Receptor signaling and genetic lesions in TP53 and CDKN2A/CDKN2B cooperate in Richter Transformation. 2021.

22. Shen H, Guo M, Wang L, Cui X: MUC16 facilitates cervical cancer progression via JAK2/STAT3 phosphorylation-mediated cyclooxygenase-2 expression. Genes \& genomics 2020, 42(2):127-133.

23. Odnokoz O, Wavelet-Vermuse C, Hophan S, Bulun S, Wan YJE: ARID1 proteins: from transcriptional and post-translational regulation to carcinogenesis and potential therapeutics. 2021.

24. Stockwell BR, Friedmann Angeli JP, Bayir H, Bush Al, Conrad M, Dixon SJ, Fulda S, Gascón S, Hatzios SK, Kagan VE et al: Ferroptosis: A Regulated Cell Death Nexus Linking Metabolism, Redox Biology, and Disease. Cell 2017, 171(2):273-285.

25. Alvarez SW, Sviderskiy VO, Terzi EM, Papagiannakopoulos T, Moreira AL, Adams S, Sabatini DM, Birsoy K, Possemato R: NFS1 undergoes positive selection in lung tumours and protects cells from ferroptosis. Nature 2017, 551(7682):639-643.

26. Liang C, Zhang X, Yang M, Dong XJAm: Recent Progress in Ferroptosis Inducers for Cancer Therapy. 2019, 31(51):e1904197.

27. Kanai Y, Hediger MJPAEjop: The glutamate/neutral amino acid transporter family SLC1: molecular, physiological and pharmacological aspects. 2004, 447(5):469-479.

28. Nicklin P, Bergman P, Zhang B, Triantafellow E, Wang H, Nyfeler B, Yang H, Hild M, Kung C, Wilson C et al: Bidirectional transport of amino acids regulates mTOR and autophagy. 2009, 136(3):521-534.

29. Hassanein M, Hoeksema M, Shiota M, Qian J, Harris B, Chen H, Clark J, Alborn W, Eisenberg R, Massion PJCcraojotAAfCR: SLC1A5 mediates glutamine transport required for lung cancer cell growth and survival. 2013, 19(3):560-570.

30. Wang Q, Beaumont KA, Otte NJ, Font J, Bailey CG, van Geldermalsen M, Sharp DM, Tiffen JC, Ryan $\mathrm{RM}$, Jormakka $\mathrm{M}$ et al: Targeting glutamine transport to suppress melanoma cell growth. International journal of cancer 2014, 135(5):1060-1071.

31. Ren P, Yue M, Xiao D, Xiu R, Gan L, Liu H, Qing G: ATF4 and N-Myc coordinate glutamine metabolism in MYCN-amplified neuroblastoma cells through ASCT2 activation. The Journal of pathology 2015, 235(1):90-100.

32. Niu Y, Zhang J, Tong Y, Li J, Liu BJLs: Physcion 8-O-ß-glucopyranoside induced ferroptosis via regulating miR-103a-3p/GLS2 axis in gastric cancer. 2019, 237:116893.

33. Luo M, Wu L, Zhang K, Wang H, Zhang T, Gutierrez L, O'Connell D, Zhang P, Li Y, Gao T et al: miR-137 regulates ferroptosis by targeting glutamine transporter SLC1A5 in melanoma. Cell Death Differ 2018, 25(8):1457-1472.

34. Hato $\mathrm{T}$, Tabata $\mathrm{M}$, Oike $\mathrm{Y}$ : The role of angiopoietin-like proteins in angiogenesis and metabolism. Trends in cardiovascular medicine 2008, 18(1):6-14.

35. Baba K, Kitajima Y, Miyake S, Nakamura J, Wakiyama K, Sato H, Okuyama K, Kitagawa H, Tanaka T, Hiraki $\mathrm{M}$ et al: Hypoxia-induced ANGPTL4 sustains tumour growth and anoikis resistance through different mechanisms in scirrhous gastric cancer cell lines. Scientific reports 2017, 7(1):11127. 
36. Liao YH, Chiang KH, Shieh JM, Huang CR, Shen CJ, Huang WC, Chen BK: Epidermal growth factorinduced ANGPTL4 enhances anoikis resistance and tumour metastasis in head and neck squamous cell carcinoma. Oncogene 2017, 36(16):2228-2242.

37. Yang W-H, Huang Z, Wu J, Ding C-KC, Murphy SK, Chi J-T: A TAZ-ANGPTL4-NOX2 Axis Regulates Ferroptotic Cell Death and Chemoresistance in Epithelial Ovarian Cancer. Mol Cancer Res 2020, 18(1):79-90.

38. Wang H, Hu S, Chen X, Shi H, Chen C, Sun L, Chen ZJ: cGAS is essential for the antitumor effect of immune checkpoint blockade. Proceedings of the National Academy of Sciences of the United States of America 2017, 114(7):1637-1642.

39. Schadt L, Sparano C, Schweiger N, Silina K, Cecconi V, Lucchiari G, Yagita H, Guggisberg E, Saba S, Nascakova Z et al: Cancer-Cell-Intrinsic cGAS Expression Mediates Tumor Immunogenicity. 2019, 29(5):1236-1248.e1237.

40. Liu J, Dai E, Kang R, Kroemer G, Tang D: The dark side of ferroptosis in pancreatic cancer. Oncoimmunology 2021, 10(1):1868691-1868691.

41. Gambardella V, Castillo J, Tarazona N, Gimeno-Valiente F, Fleitas TJCTR: The role of TumorAssociated Macrophages in Gastric Cancer development and their potential as a therapeutic target. 2020, 86:102015.

42. Gu-Trantien C, Loi S, Garaud S, Equeter C, Willard-Gallo KJJoCl: CD4+ follicular helper T cell infiltration predicts breast cancer survival. 2013, 123(7):2873-2892.

43. Jiang L, Kon N, Li T, Wang S-J, Su T, Hibshoosh H, Baer R, Gu W: Ferroptosis as a p53-mediated activity during tumour suppression. Nature 2015, 520(7545):57-62.

44. Yang W, SriRamaratnam R, Welsch M, Shimada K, Skouta R, Viswanathan V, Cheah J, Clemons P, Shamji A, Clish C et al: Regulation of ferroptotic cancer cell death by GPX4. 2014, 156:317-331.

45. Chang W-T, Bow Y-D, Fu P-J, Li C-Y, Wu C-Y, Chang Y-H, Teng Y-N, Li R-N, Lu M-C, Liu Y-C et al: A Marine Terpenoid, Heteronemin, Induces Both the Apoptosis and Ferroptosis of Hepatocellular Carcinoma Cells and Involves the ROS and MAPK Pathways. Oxid Med Cell Longev 2021, 2021:7689045-7689045.

46. Poursaitidis I, Wang X, Crighton T, Labuschagne C, Mason D, Cramer SL, Triplett K, Roy R, Pardo OE, Seckl MJ et al: Oncogene-Selective Sensitivity to Synchronous Cell Death following Modulation of the Amino Acid Nutrient Cystine. Cell reports 2017, 18(11):2547-2556.

\section{Figures}




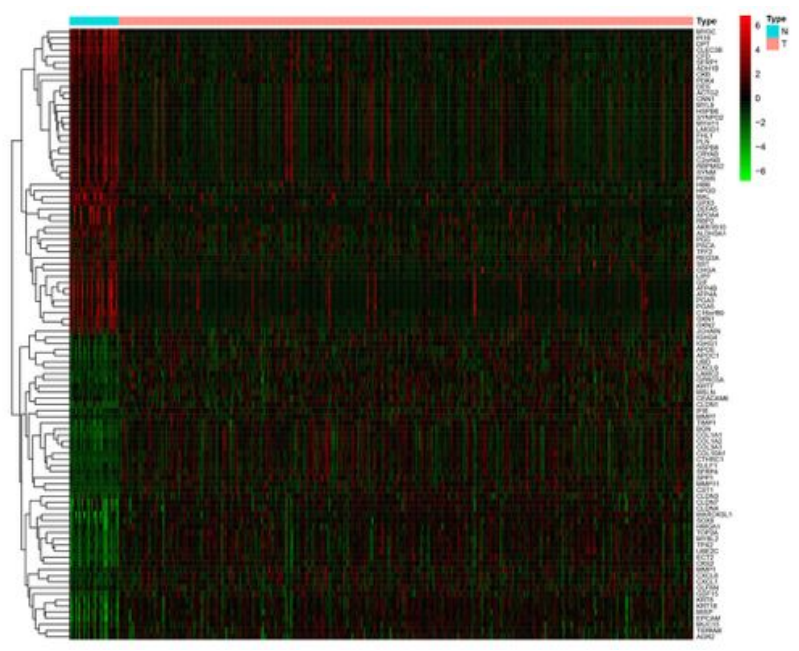

b

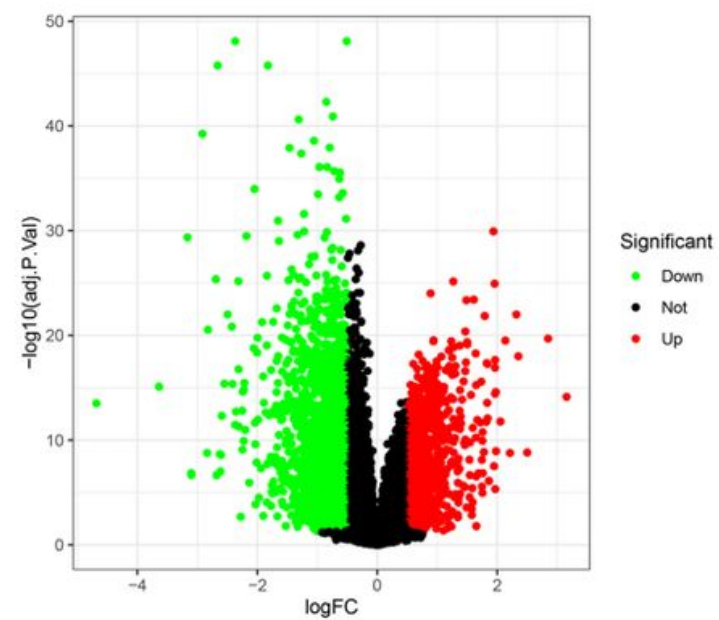

\section{Figure 1}

Differentially expressed genes (DEGs) obtained. a The heatmap of DEGs expression level in GC samples. b The volcano plot of DEGs in GC. Red and blue indicate up-regulation and down-regulation, respectively. $\mathrm{N}$, normal sample; T, tumor tissues. 


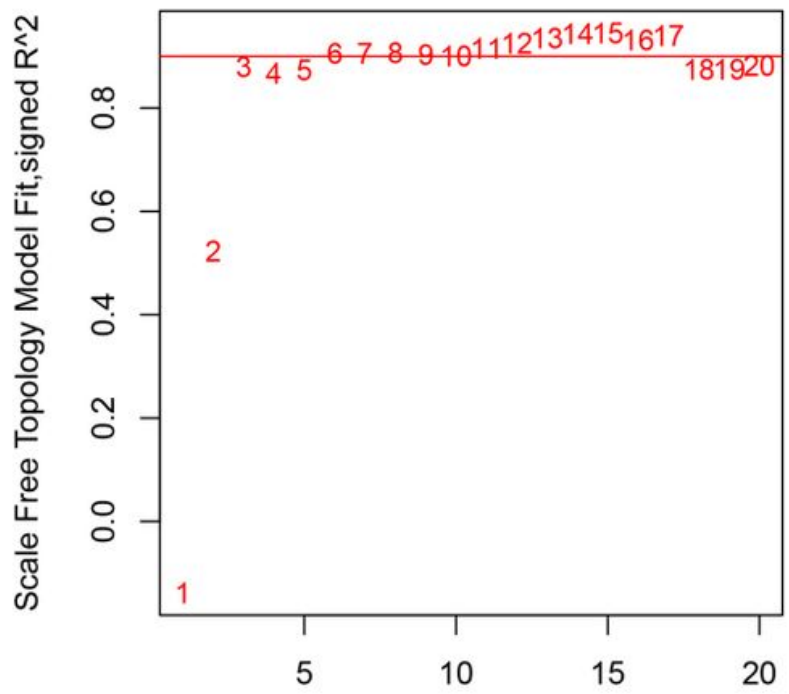

Soft Threshold (power)

b

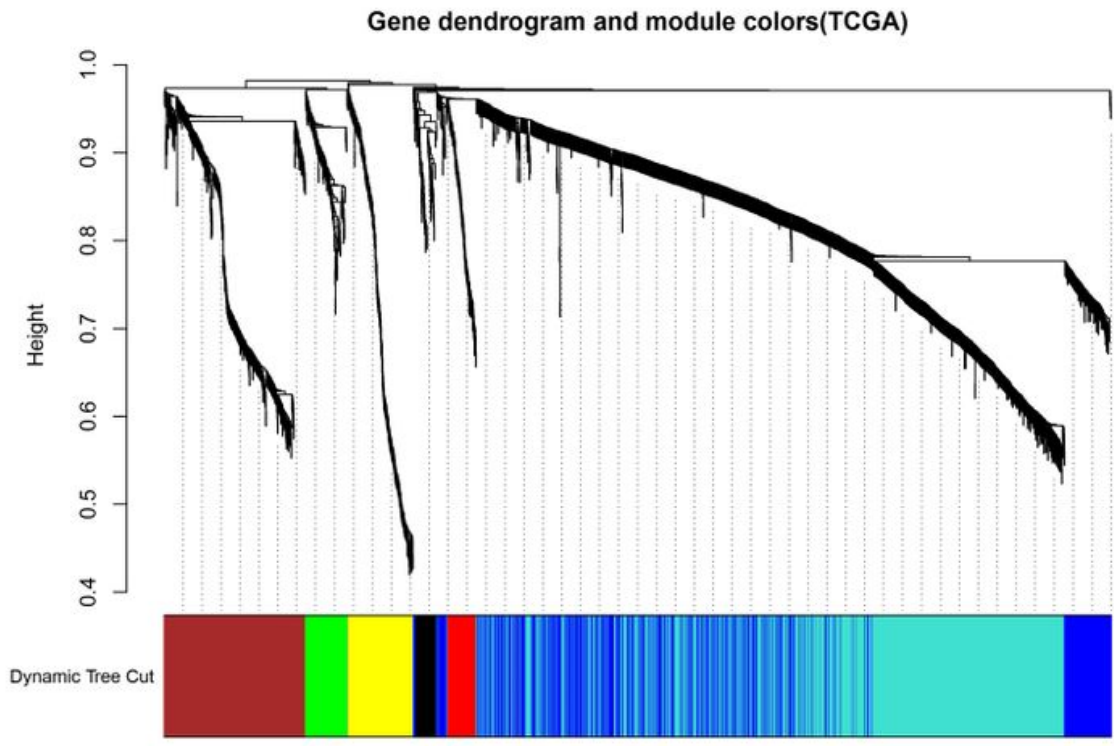

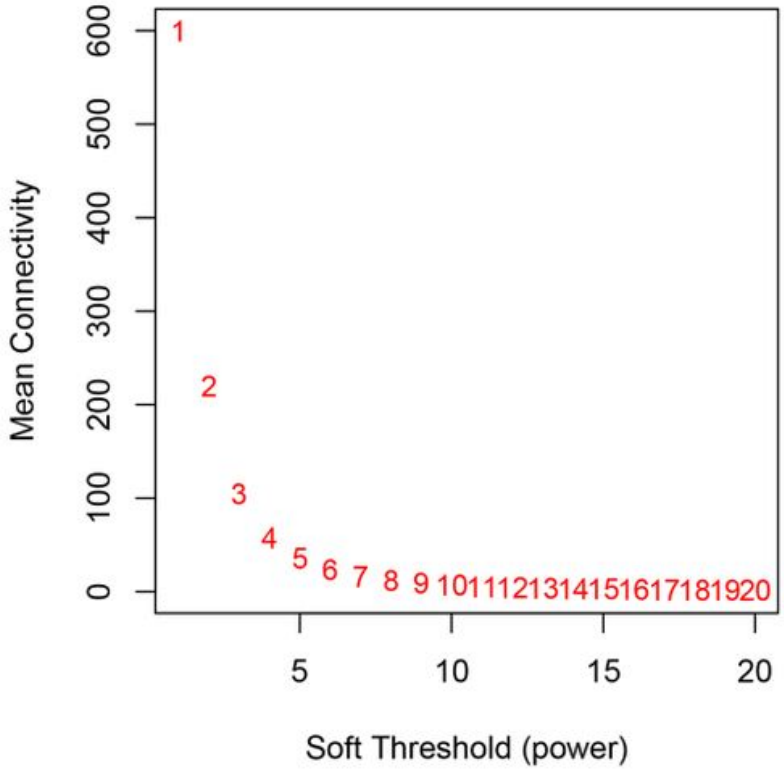

$\mathrm{C}$

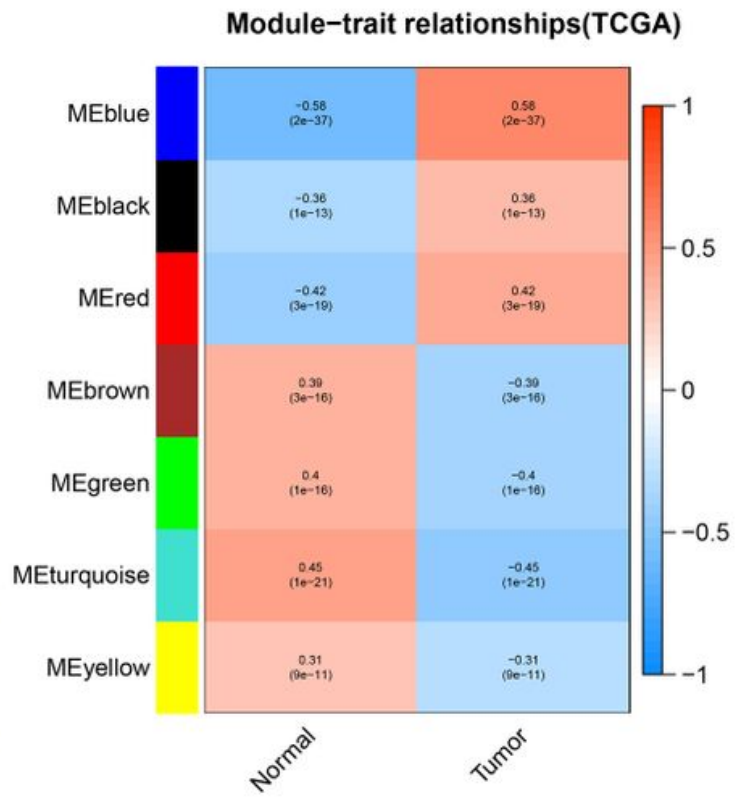

\section{Figure 2}

Identification of GC-related module genes by WGCNA analysis. a The optimal power was 3 when the red guide line was set to 0.89 . The mean connectivity of different soft-threshold powers. $b$ Clustering of dendrogram and corresponding modules. c Heatmap showing the correlation of gene modules with GC samples or normal samples. 
a

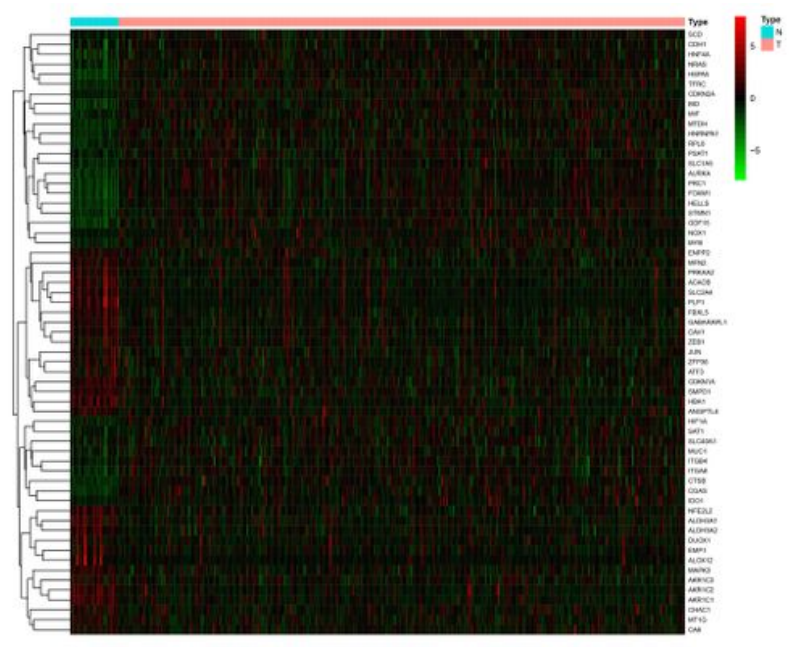

$\mathrm{b}$

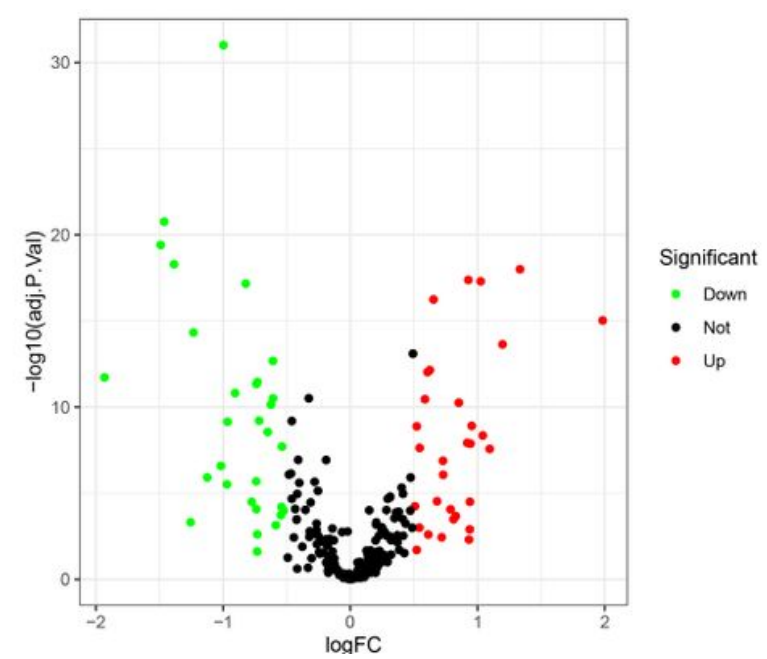

\section{Figure 3}

Differentially expressed FRGs (DEFRGs) obtained. a The heatmap of DEFRGs expression level in GC samples. $b$ The volcano plot of DEFRGs in GC. Red and blue indicate up-regulation and down-regulation, respectively. N, normal sample; T, tumor tissues.

a

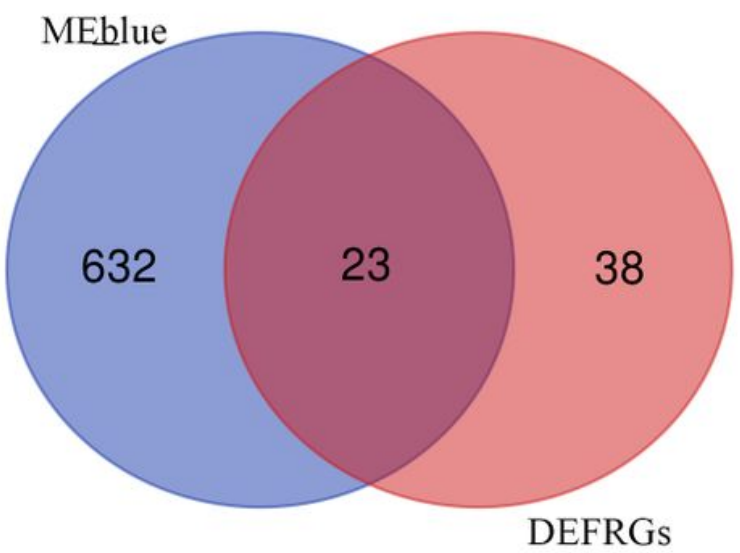

b

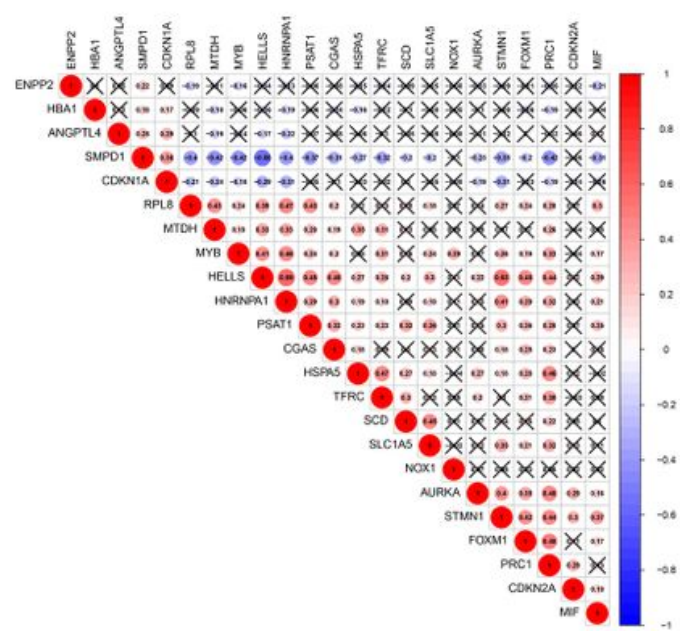

Figure 4

a Venn diagram displaying the number of genes in different groups. b Pearson correlation analysis shows relationship between the 23 FRGs based on their expression in GC tissues. 

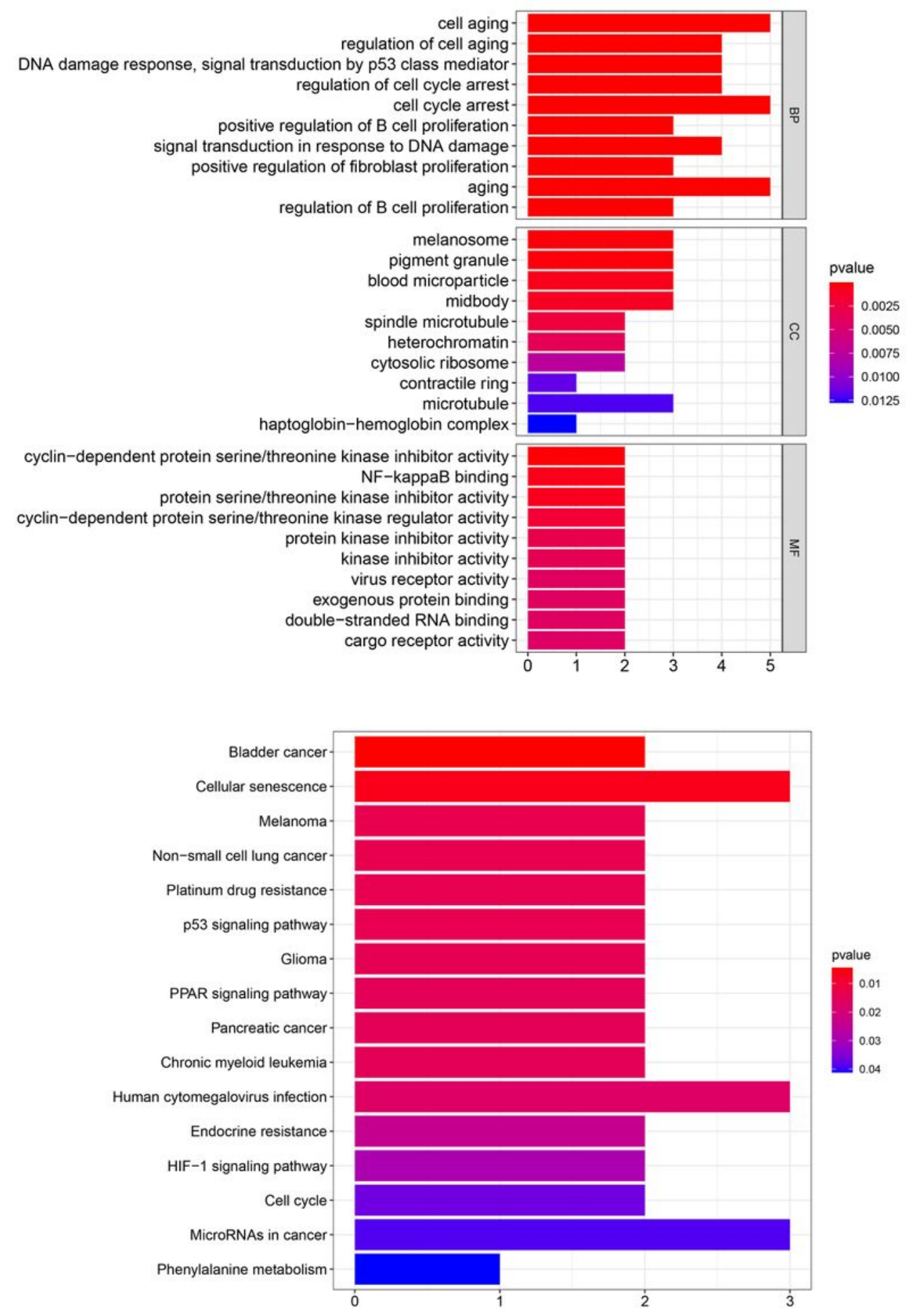

\section{Figure 5}

Gene functional annotation of the set of 23 shared genes. a GO analysis of the 23 shared genes including $\mathrm{BP}, \mathrm{CC}$, and MF. b KEGG pathway enrichment analysis of the 23 shared genes. $\mathrm{P}<0.05$. 
pvalue

Hazard ratio

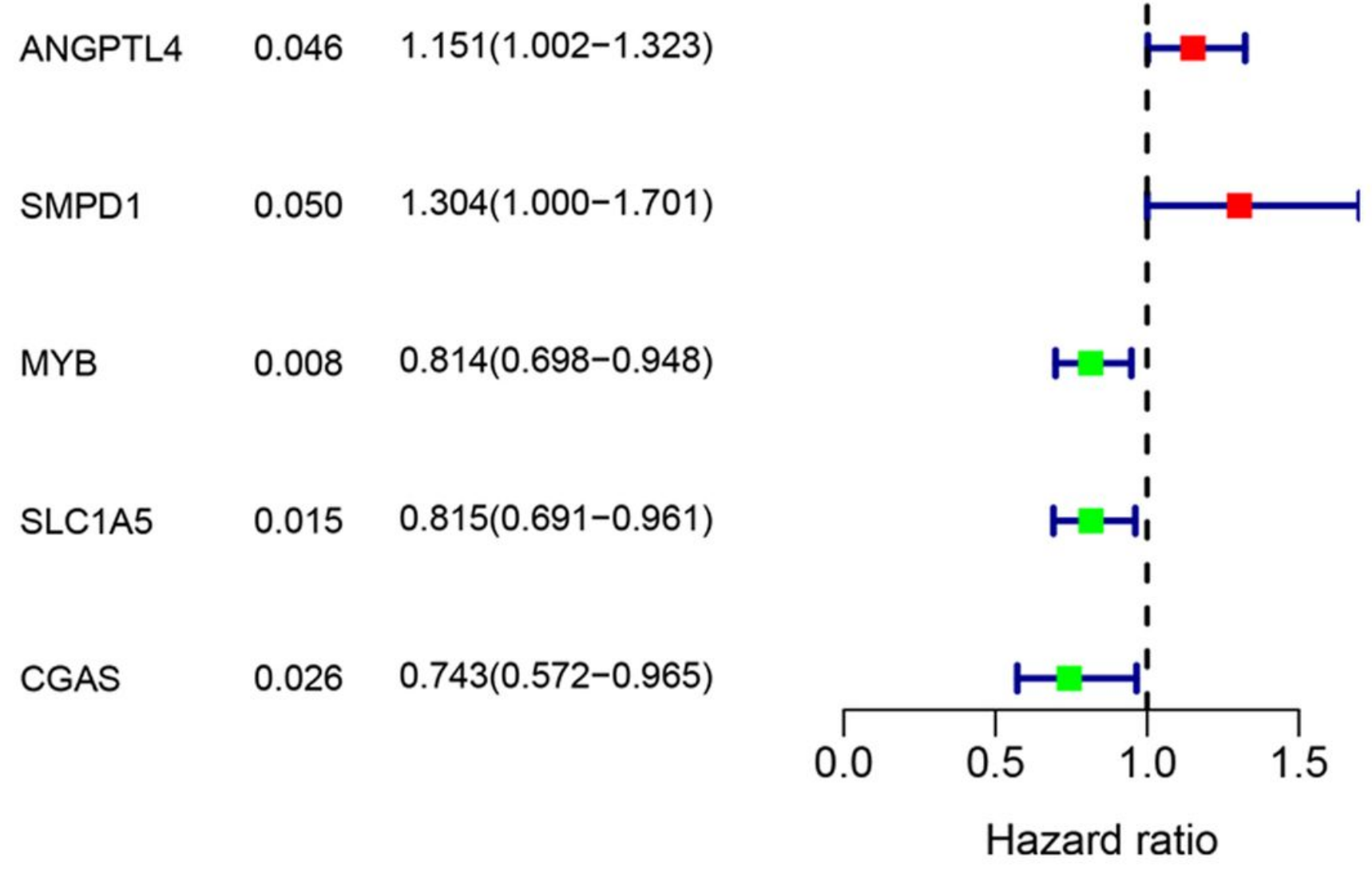

Figure 6

Screening prognostic factors through UCR analysis. 
a

\section{TCGA dataset}

Risk + High risk — Low risk
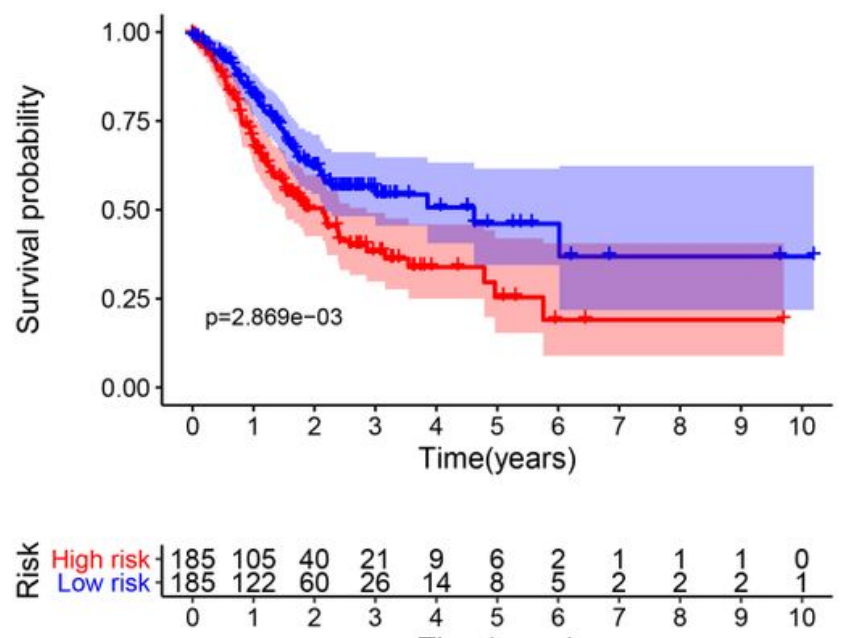

b

Time(years)

c

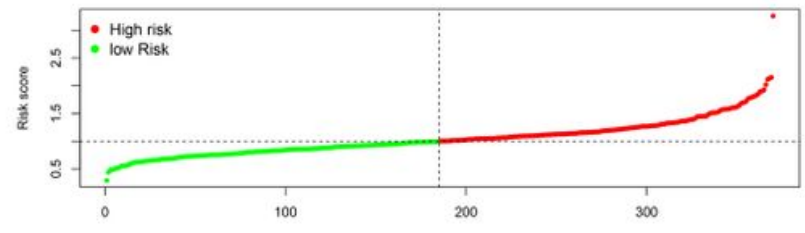

Patients (ncreasing risk socre)

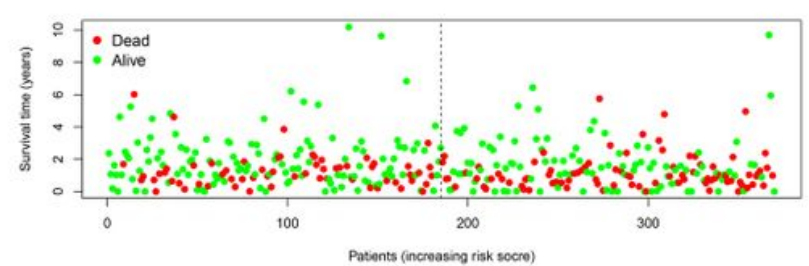

d

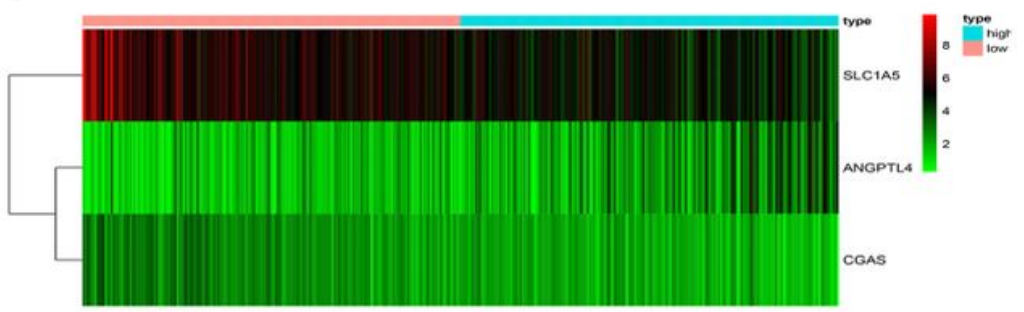

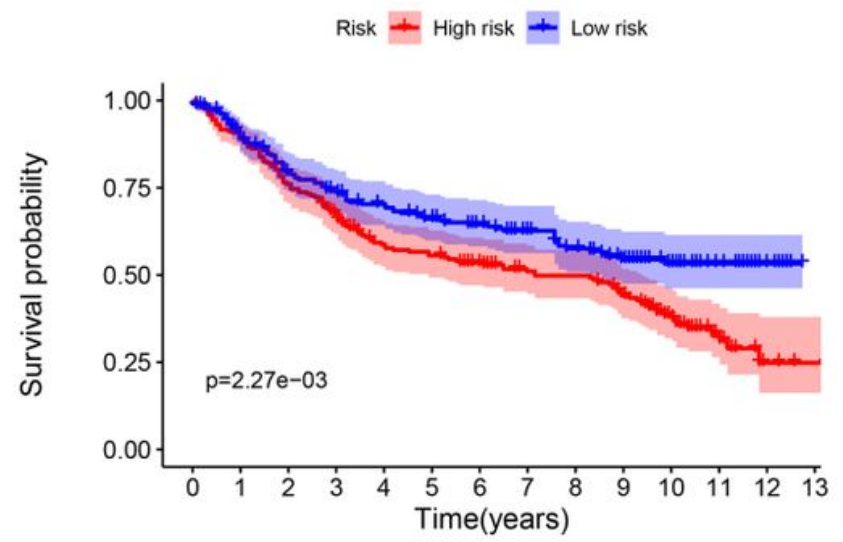

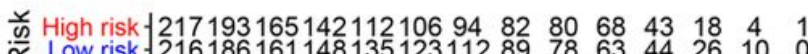

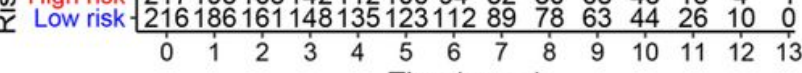
f
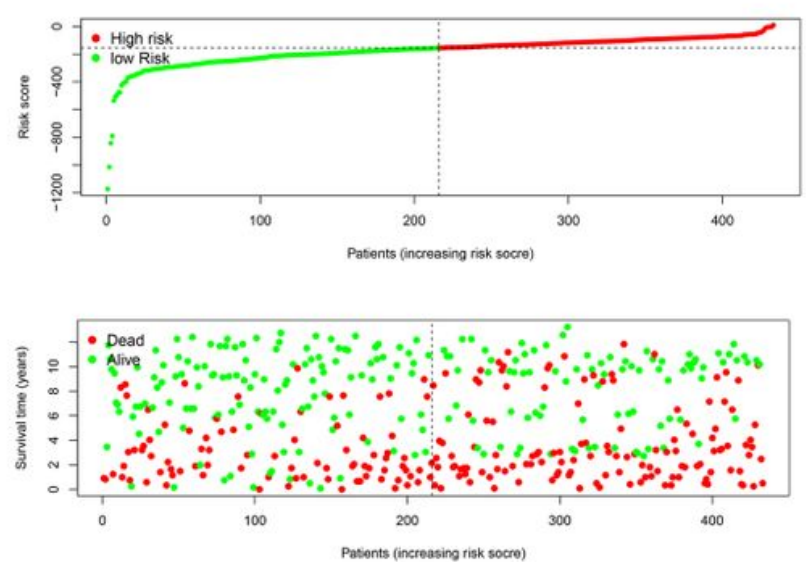

$\mathrm{h}$

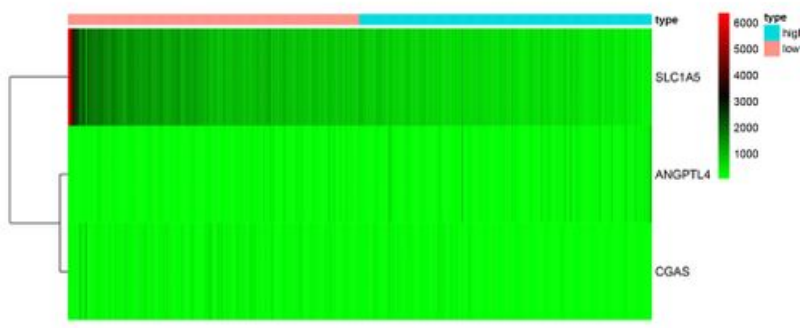

Figure 7

Prognostic model based on FRGs in GC. K-M survival analysis a, risk score distribution b, survival status C and heatmap $\mathrm{d}$ of a prognostic model in the GC cohort from TCGA. K-M survival analysis e, risk score distribution $\mathrm{f}$, survival status $\mathrm{g}$ and heatmap $\mathrm{h}$ of a prognostic model in the GC cohort from GEO. 


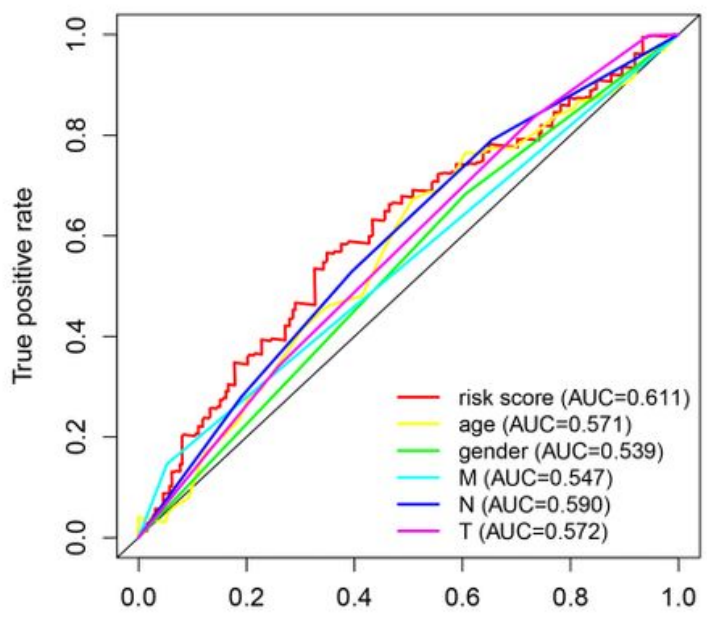

b

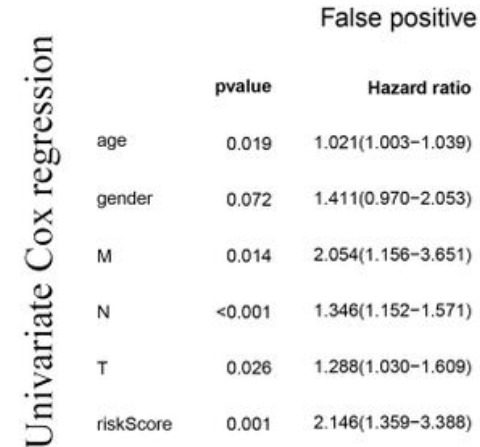

$\mathrm{c}$

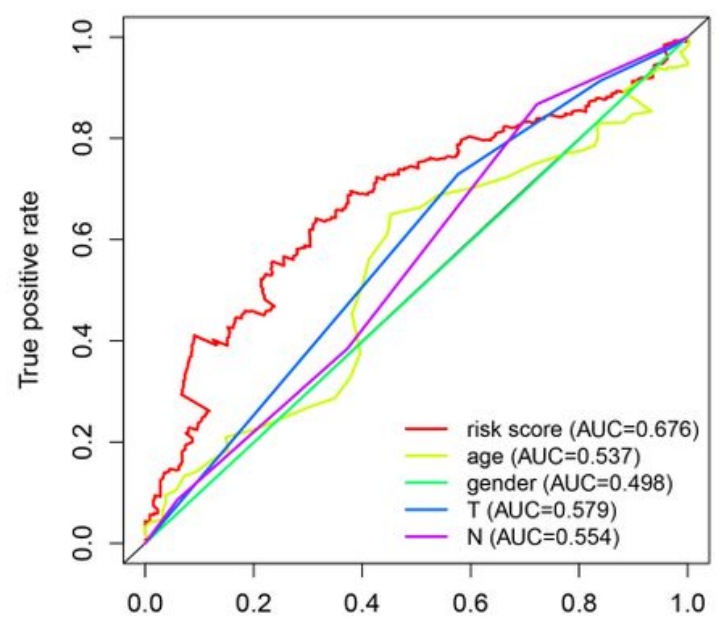

$\mathrm{e}$

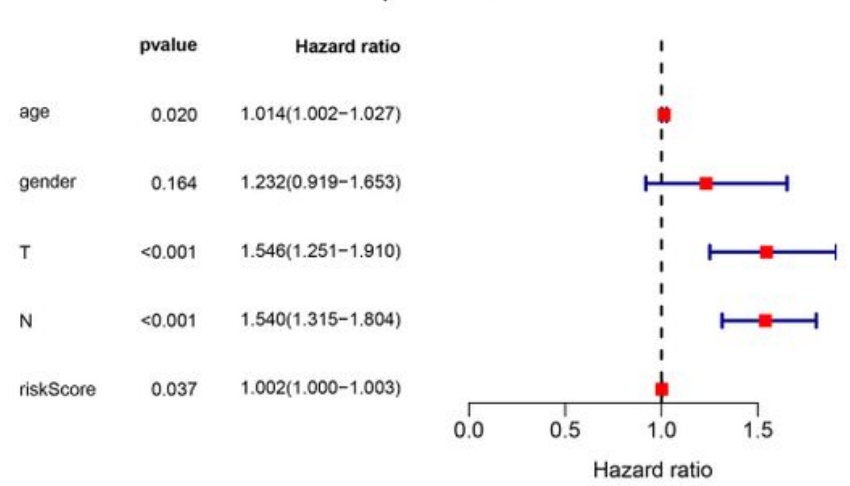

$\mathrm{f}$

False positive rate
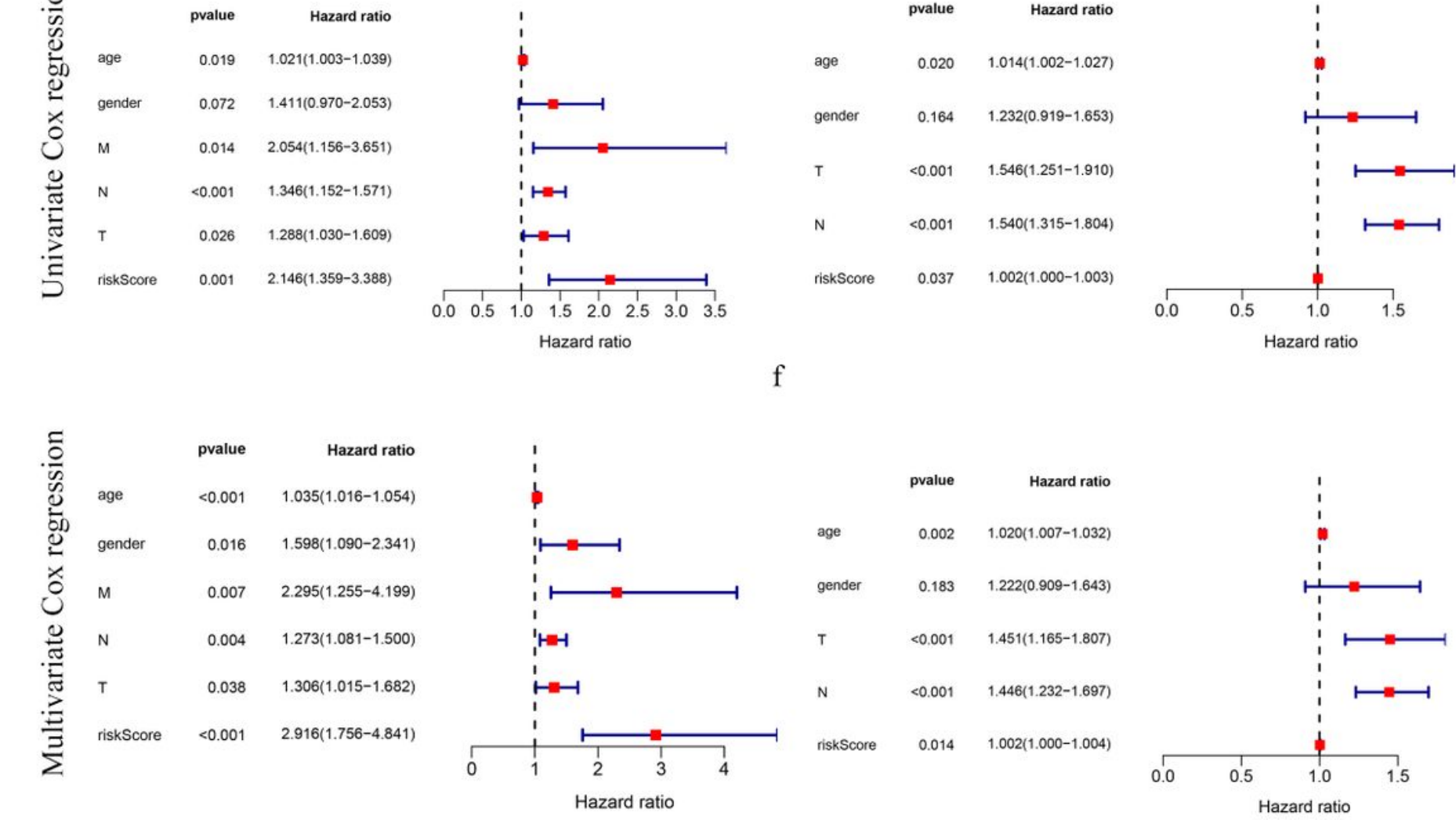

Figure 8

Prognostic value of risk subgroups in TCGA and GEO dataset. ROC curves $a$, UCR b and MCR $c$ analysis of the risk score and other clinical indices in TCGA cohort. ROC curves $d$, UCR e and MCR $f$ analysis of the risk score and other clinical indices in GEO cohort. 


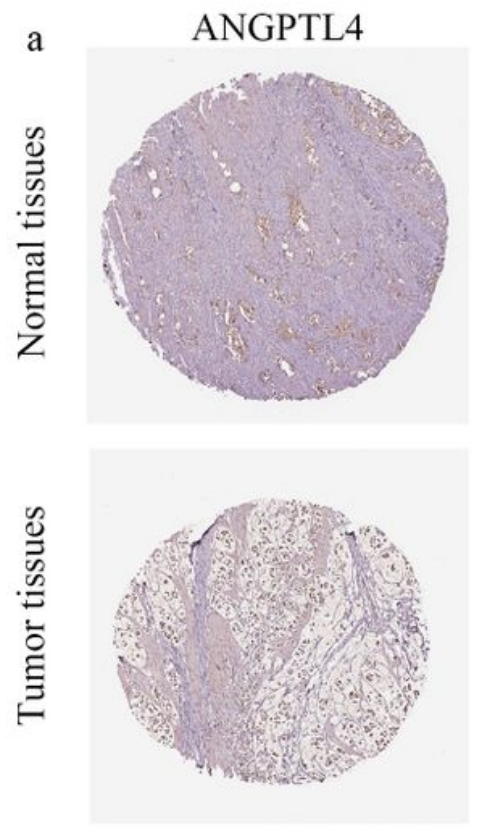

$\mathrm{b}$
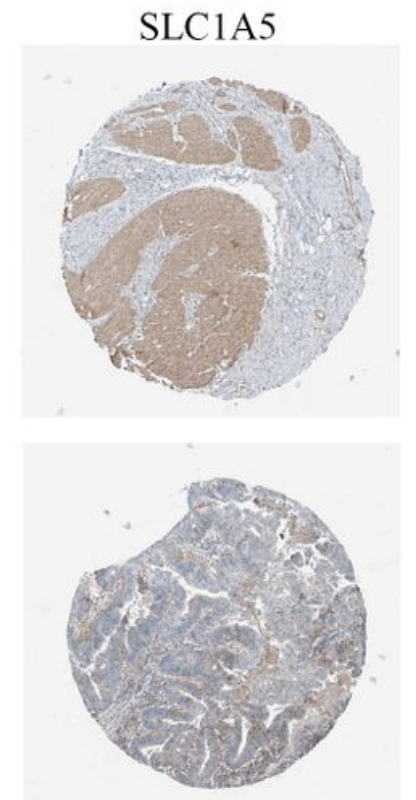

$\mathrm{c}$

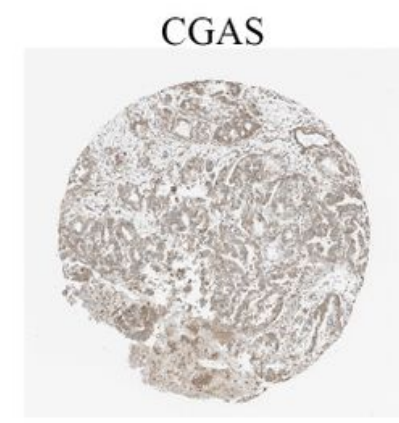

\section{Figure 9}

The expression level of three genes in GC tissues. a ANGPTL4, b SLC1A5, and c CGAS

a

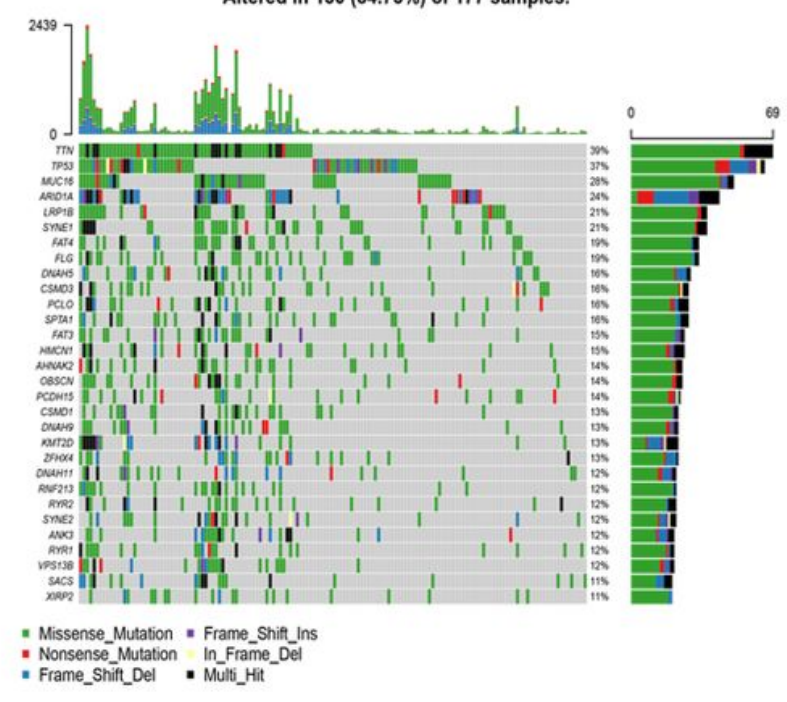

$\mathrm{b}$

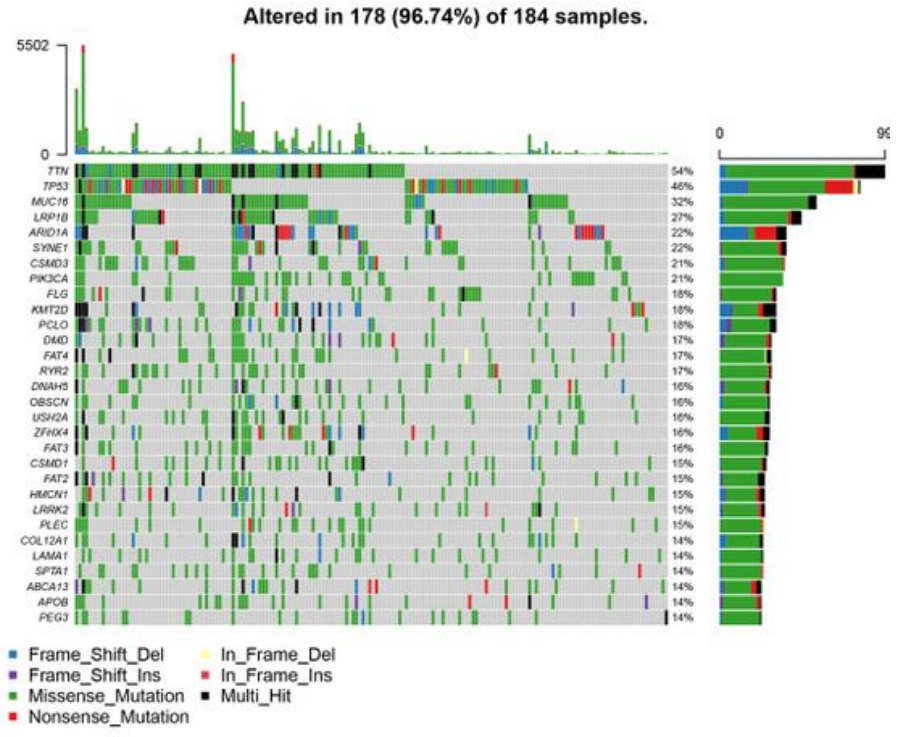

Figure 10

Genomic mutation profiles of different risk groups. Low risk group a, high risk group b. 
Risk 追 low 追 high

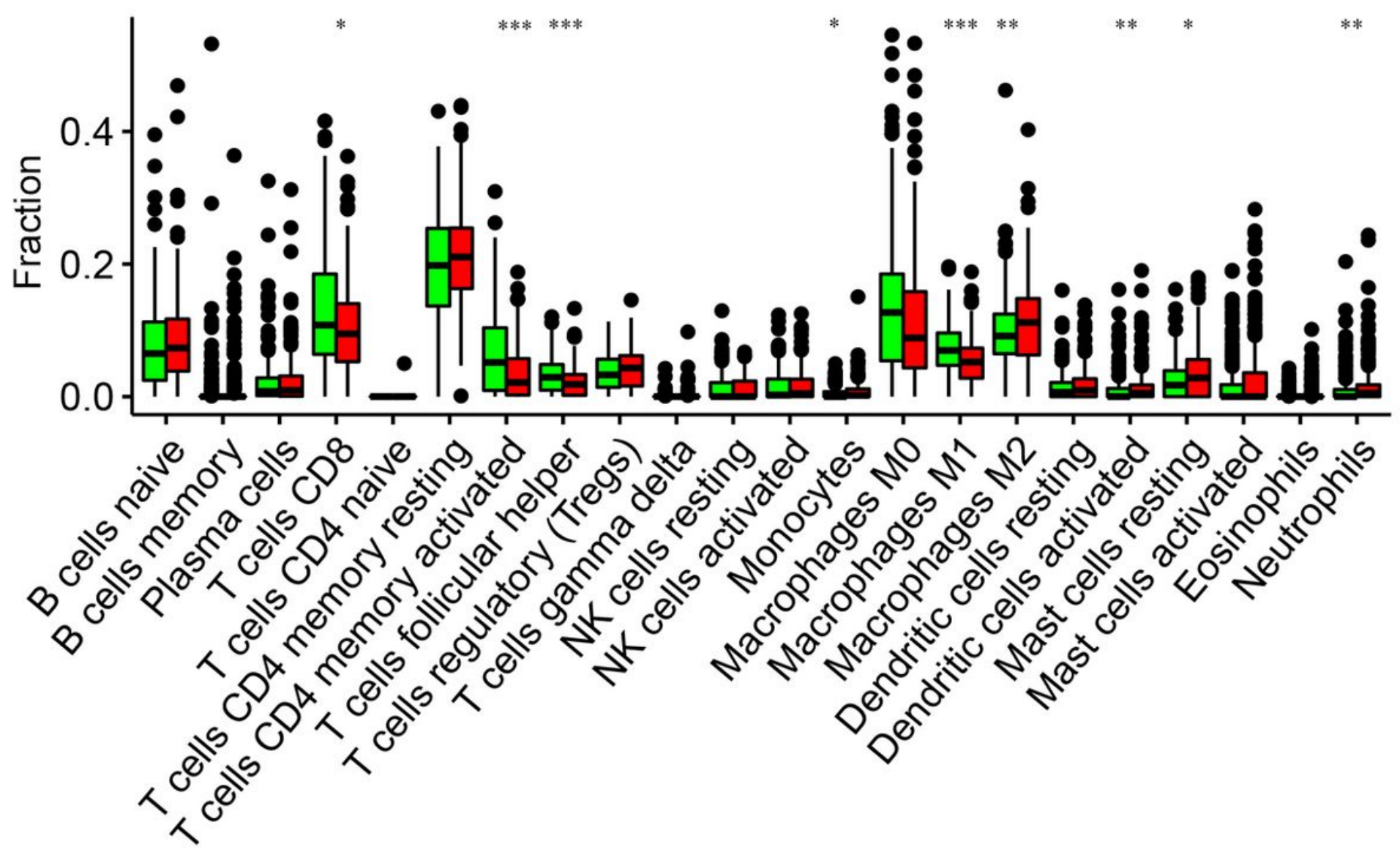

Figure 11

Relative proportion of immune infiltration in high- and low-risk patients of the TCGA cohort. ${ }^{\star} p<0.05$; ${ }^{* \star} p$ $<0.01 ; * \star \star p<0.005$ 


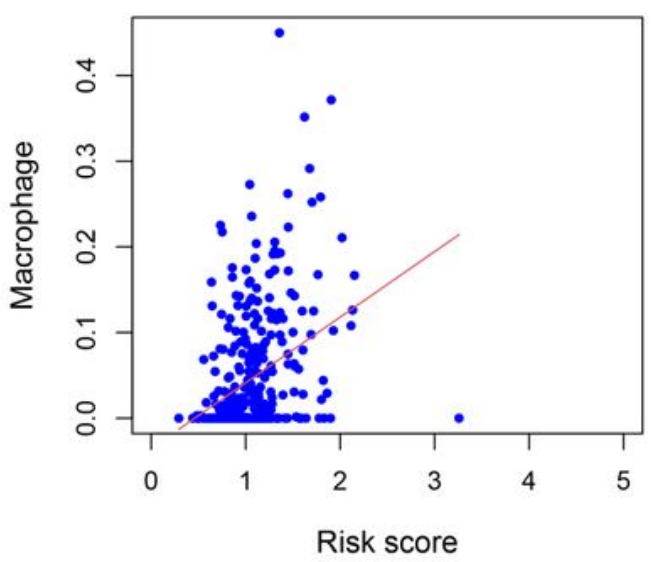

c

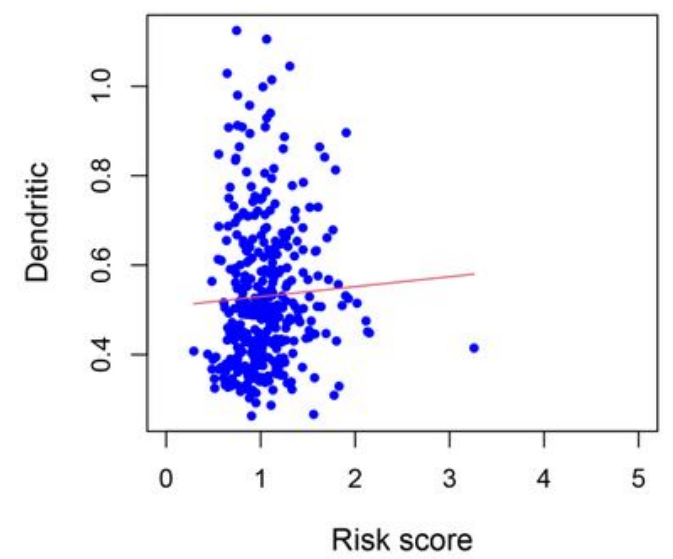

e Cor $=-0.076(p=0.151)$

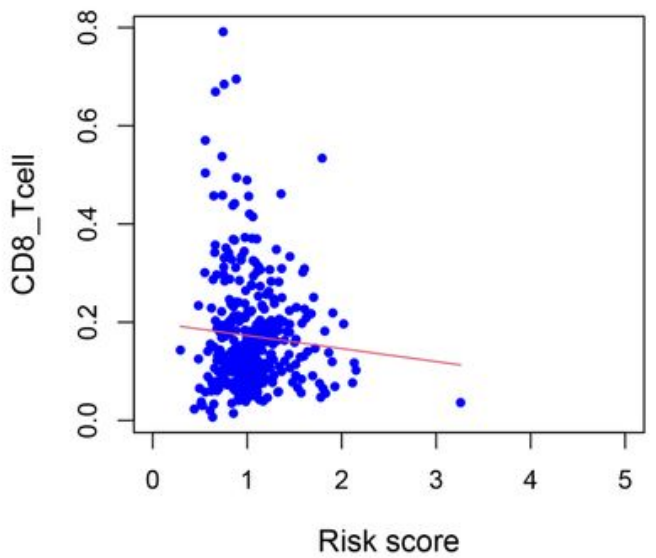

b Cor $=0.027(p=0.615)$

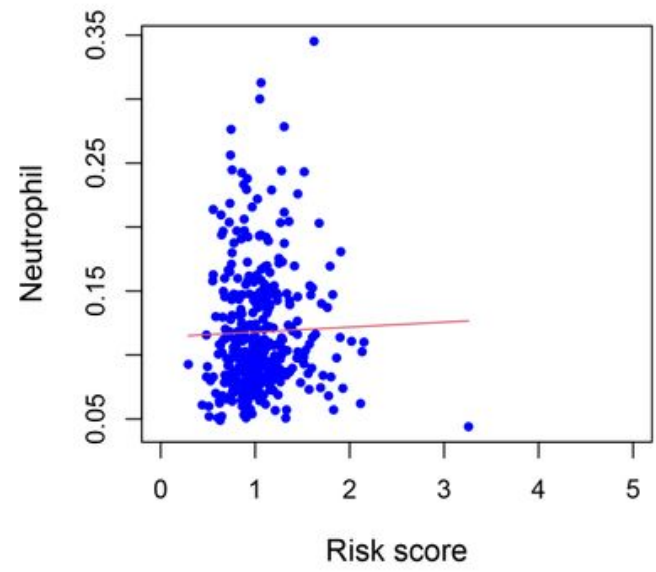

d

Cor $=-0.076(p=0.151)$

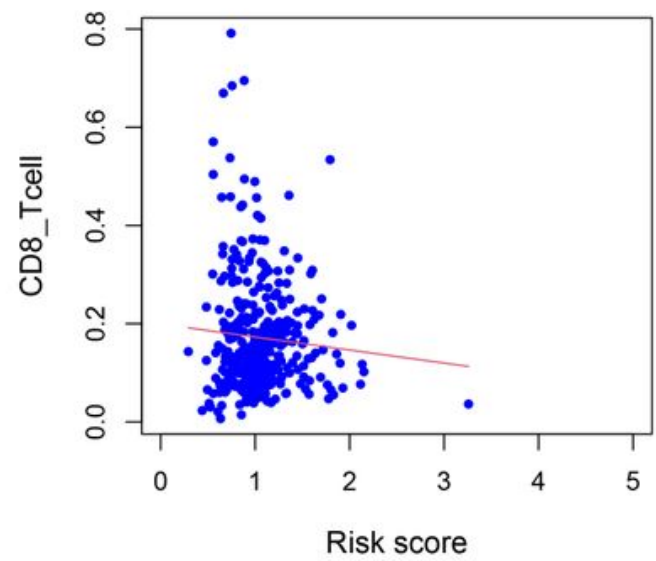

f

Cor=0.135 $(p=0.010)$

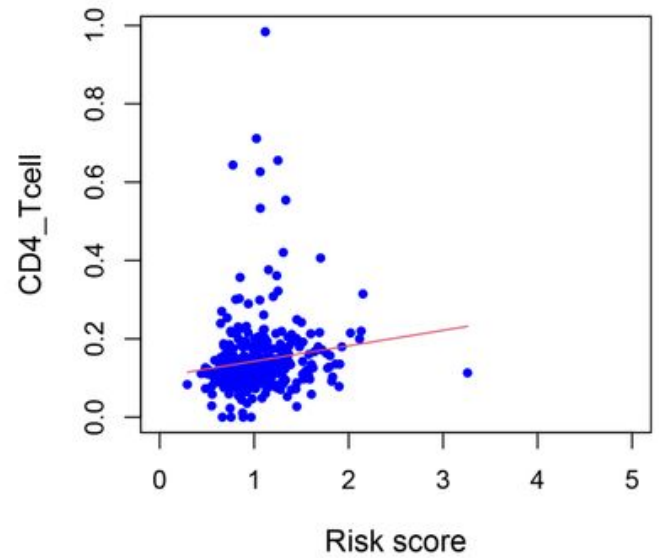

Figure 12

Correlation analysis between risk scores and immune cell infiltrations. 

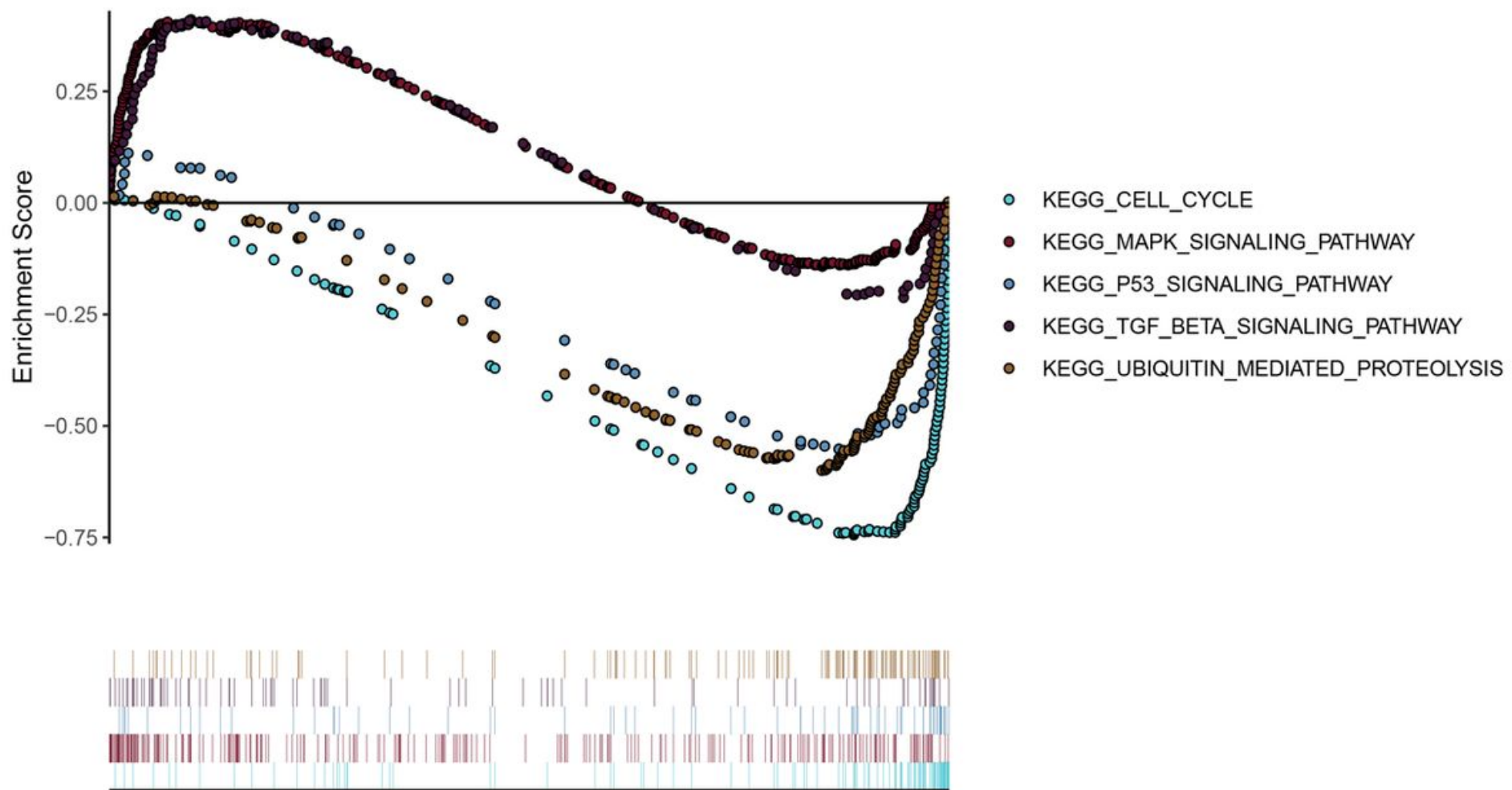

High risk<-----------> Low risk

Figure 13

Functional enrichment analyses regarding the risk score. 